\title{
Exciton lifetimes of CdTe nanocrystal quantum dots in high magnetic fields
}

\author{
J. H. Blokland, ${ }^{1}$ V. I. Claessen, ${ }^{2}$ F. J. P. Wijnen, ${ }^{1}$ E. Groeneveld, ${ }^{3}$ C. de Mello Donegá, ${ }^{3}$ D. Vanmaekelbergh, ${ }^{3}$ A. Meijerink, ${ }^{3}$ \\ J. C. Maan, ${ }^{1}$ and P. C. M. Christianen ${ }^{1, *}$ \\ ${ }^{1}$ Radboud University Nijmegen, Institute for Molecules and Materials, High Field Magnet Laboratory, NL-6525 ED Nijmegen, \\ The Netherlands \\ ${ }^{2}$ Radboud University Nijmegen, Institute for Molecules and Materials, NL-6525 ED Nijmegen, The Netherlands \\ ${ }^{3}$ Debye Institute, Condensed Matter and Interfaces, Utrecht University, P.O. Box 80 000, NL-3508 TA Utrecht, The Netherlands
}

(Received 8 April 2010; published 11 January 2011)

\begin{abstract}
We have measured the low-temperature $(4.2 \mathrm{~K})$ exciton lifetimes of zinc-blende CdTe nanocrystal quantum dots (NQDs), 2.6-3.8 $\mathrm{nm}$ in diameter, in magnetic fields up to $30 \mathrm{~T}$. The exciton photoluminescence decay time decreases with both dot size and magnetic field. We explain the decrease in decay time in magnetic fields by the mixing of bright and dark exciton states due to a small shape asymmetry in the zinc-blende CdTe NQDs. We show that this behavior resembles that of wurtzite CdSe NQDs, and we demonstrate that an asymmetry of NQDs caused by either shape or crystal structure leads to similar exciton decay dynamics.
\end{abstract}

DOI: 10.1103/PhysRevB.83.035304

PACS number(s): 78.67.Hc, 78.47.jg, 71.35.-y

\section{INTRODUCTION}

The optical properties of colloidal semiconductor nanocrystal quantum dots (NQDs) have attracted much interest in recent years. Control over their composition and size makes it possible to tune their exciton confinement energies, and thereby their emission wavelength, over a wide range. ${ }^{1,2}$ Furthermore, the high photoluminescence (PL) quantum efficiency of high-quality NQDs at room temperature ${ }^{3,4}$ is important for applications. ${ }^{5-9}$ To date, wurtzite NQDs have been mostly investigated, with $\mathrm{CdSe}$ as the prototypical example, ${ }^{10-12}$ since the PL wavelength of CdSe NQDs can be tuned across the visible wavelength region. Alternatively, zinc-blende (e.g., CdTe, CdS, ZnSe, and $\mathrm{ZnS}$ ) and rock-salt (e.g., $\mathrm{PbS}$ and $\mathrm{PbSe}$ ) NQDs have been fabricated, which have considerably enlarged the wavelength emission range. ${ }^{13,14}$

From a fundamental point of view, it is interesting to study NQDs with different crystal structures because the lattice symmetry is one of the parameters to tune the lowest energy levels of the excitons that are responsible for the NQD optical properties. ${ }^{10,15,16}$ It is well established that, in wurtzite NQDs, the electron-hole exchange interaction and the intrinsic crystal/shape anisotropy lift the spin degeneracy of the exciton levels, leading to five distinct states, which are characterized by their spin projection $\left(F_{m}\right)$ along the nanocrystal $c$ axis. The resulting exciton fine structure is characterized by a lowest energy exciton state $\left(F_{m}= \pm 2\right)$, which is forbidden for direct radiative recombination (thus optically dark), and a higher energy exciton state $\left(F_{m}= \pm 1^{L}\right)$, which is dipole allowed (thus optically bright). Evidence for this exciton fine structure has been given by the size-dependent Stokes shift from fluorescence line-narrowing experiments ${ }^{15,17}$ and by the dependence of the radiative lifetime on both temperature ${ }^{11,18-22}$ and magnetic fields. ${ }^{11,23,24}$ In these latter experiments, it was found that the dark ground state acquires oscillator strength in a magnetic field by field-induced mixing of the exciton states, thereby significantly decreasing the exciton lifetime. These experiments demonstrate that application of high magnetic fields is a powerful tool to determine the exciton fine structure in NQDs. ${ }^{11,15,23-25}$ Similar high-field data are scarce for NQDs with a crystal lattice different from wurtzite. ${ }^{26}$
In this paper, we report on the radiative lifetimes of excitons in zinc-blende CdTe NQDs as a function of strong magnetic fields $(B)$, as determined from the PL decay times. We have found that the exciton lifetime strongly decreases with increasing magnetic field, as well as with increasing temperature and size, which is similar to the behavior of wurtzite CdSe NQDs. We describe the $B$ dependence of the exciton lifetime with a phenomenological model that includes $B$-induced exciton mixing. Based on the fact that the theoretically predicted exciton fine structure is similar for spherical NQDs with the wurtzite crystal structure and for prolate NQDs with the zinc-blende crystal structure, we conclude that the close resemblance of the CdTe and the CdSe data is caused by the nonspherical shapes of the CdTe NQDs investigated in this work.

\section{EXPERIMENT}

We have measured the magnetic-field dependence of the PL decay time for five different chemically synthesized CdTe NQD samples, with diameters $(d)$ ranging from 2.6 to $3.8 \mathrm{~nm}$, and for one CdSe NQD sample, with $d=3.6 \mathrm{~nm}$ (size dispersion $<10 \%$ in all cases). The synthesis and characterization of the NQDs have been described elsewhere. ${ }^{27,28}$ Powder $\mathrm{X}$-ray diffraction measurements revealed that the CdTe NQDs have the zinc-blende crystal structure [Fig. 1(c)]. Transmission electron microscopy showed that NQDs fabricated by this method possess a variety of faceted and nonspherical shapes, sometimes slightly elongated in one direction (aspect ratio: 1.1-1.2) [Figs. 1(a) and 1(b)]. The samples were prepared by freezing a suspension of randomly organized, organically capped NQDs in toluene. Time-correlated singlephoton counting experiments were performed in high magnetic fields up to $30 \mathrm{~T}$ (using a Florida-Bitter magnet in Faraday geometry) at low temperatures $(4.2 \mathrm{~K})$, using a standard PL setup with optical fibers. The excitation was provided by a picosecond pulsed laser operating at $445 \mathrm{~nm}$ (PicoQuant). The PL emission was passed through a high pass filter and detected by an avalanche photon detector (MPD PD5CTC) connected to a single-photon counter (Picoharp 300). 

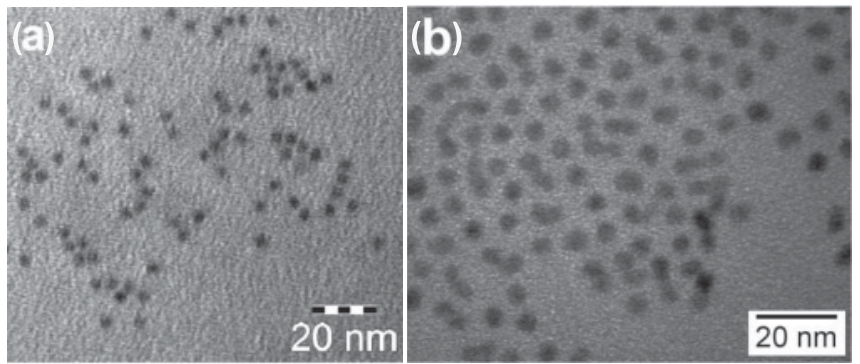

(c) CdTe NQD d= $2.6 \mathrm{~nm}$

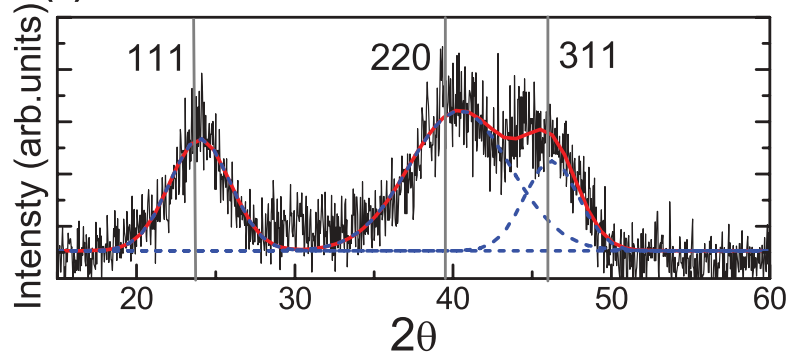

FIG. 1. (Color online) Low-resolution transmission electron microscopy images of two representative CdTe NQDs with a diameter of (a) $3.5 \mathrm{~nm}$ and (b) $5.0 \mathrm{~nm}$. (c) The x-ray powder diffraction pattern of a 2.6-nm-diameter CdTe NQD. The signal can be described (red solid line) by three peaks (blue dashed lines) at the 111, 220, and 311 maxima of the bulk zinc-blende crystal lattice.

Temperature-dependent experiments without applied magnetic field were performed using a similar setup, which was described elsewhere. ${ }^{21,22}$

\section{RESULTS}

Figure 2 shows typical PL decay curves as a function of temperature [Fig. 2(a)], NQD size [Fig. 2(b)], and magnetic field [Fig. 2(c)]. The data are background corrected and plotted on a semilogarithmic scale. The decay of all the NQDs we have investigated is biexponential, with a fast $(\approx n s)$ and a slow component. The biexponential shape indicates the high quality of the NQDs used in this study and also that interdot energy transfer plays a negligible role. The fast component is assigned to emission from the bright state prior to relaxation to the lowest energy exciton state. The decay time is determined by the radiative decay time $(\approx n s)$ for the bright state and the relaxation time to the dark state. The slow decay time reflects the lifetime for the lowest energy exciton state (the dark state). ${ }^{11,18,21,23}$ Figure 2 demonstrates that this slow decay time decreases significantly by an increasing temperature, an increment of the size and the application of a strong magnetic field.

The full magnetic-field, size, and temperature dependence of the exciton lifetimes of the NQDs are shown in Fig. 3. The data points have been obtained by fitting the slow part of the PL decay curves (from 100 to 1000 ns) by a single exponent. Figure 3(a) shows the results for the five CdTe NQDs used and demonstrates that the exciton lifetimes shorten with $B$, similar to the behavior of the reference CdSe NQD, shown in Fig. 3(b). Figure 3(a) also shows that the exciton lifetime decreases with increasing diameter, despite the deviation from this trend for the NQDs with $d=2.9-3.3 \mathrm{~nm}$. Finally, Fig. 3(c)

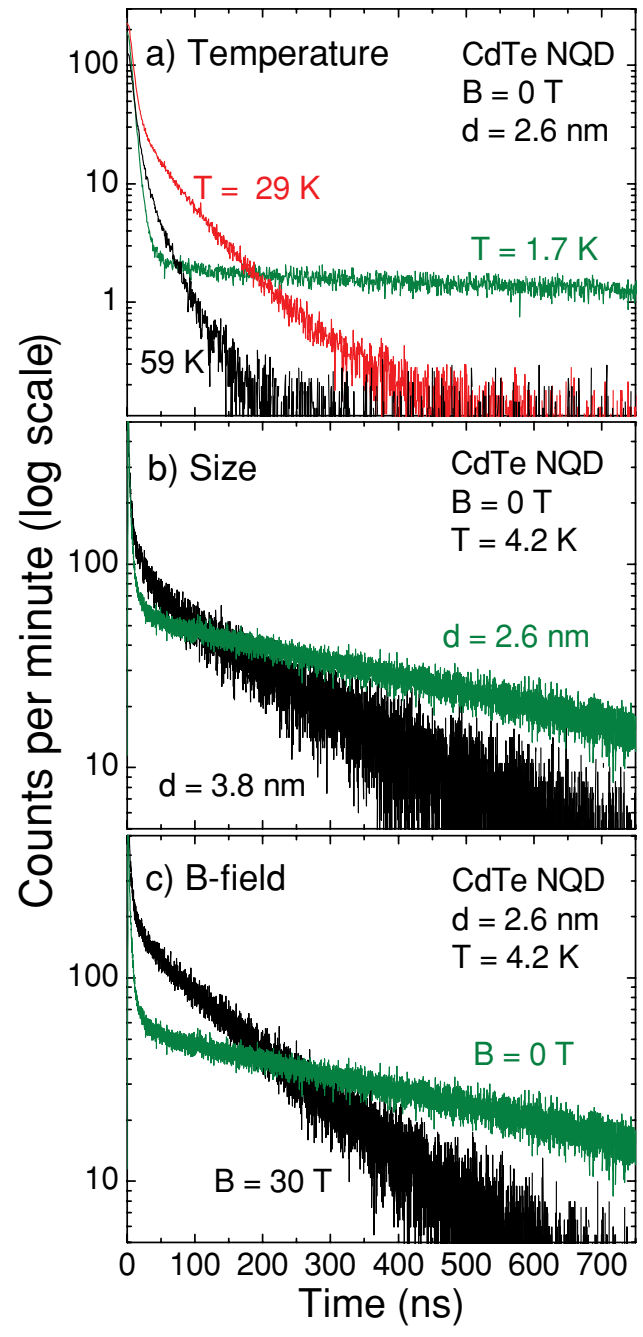

FIG. 2. (Color online) (a) Photoluminescence decay curves for a 2.6-nm-diameter CdTe NQD at $T=1.7 \mathrm{~K}, 29 \mathrm{~K}$, and $59 \mathrm{~K}$ at $B=0 \mathrm{~T}$. (b) Photoluminescence decay curves for CdTe NQDs with 3.8-nm and 2.6-nm diameters at $T=4.2 \mathrm{~K}$ and $B=0 \mathrm{~T}$. (c) Photoluminescence decay curves for a 2.6-nm diameter CdTe NQD at $B=0 \mathrm{~T}$ (grey) and $B=30 \mathrm{~T}$ (black) and $T=4.2 \mathrm{~K}$. All curves are shown on a semilogarithmic scale.

shows that the exciton lifetime of the 2.6-nm-diameter CdTe NQD decreases with increasing temperature. Below $4 \mathrm{~K}$ (the temperature used for the $B$-dependent measurements), the lifetime is constant and equal to the lifetime of the dark exciton ground state. This overall size- and temperature-dependent behavior is consistent with earlier reports ${ }^{19,22,29}$ for CdTe NQDs at $B=0$, and is remarkably similar to the behavior of CdSe NQDs. ${ }^{11,18,20-22}$

\section{DISCUSSION}

To explain the behavior of the exciton decay times, we take a closer look at the exciton fine structure of both CdSe and CdTe NQDs, as shown in Fig. 4. The energy levels have been calculated using the effective-mass model of Efros ${ }^{15}$ and depend strongly on the radius $a=d / 2$ and the crystal structure of the NQDs. In these calculations, we have used the following parameters: the exchange splitting 


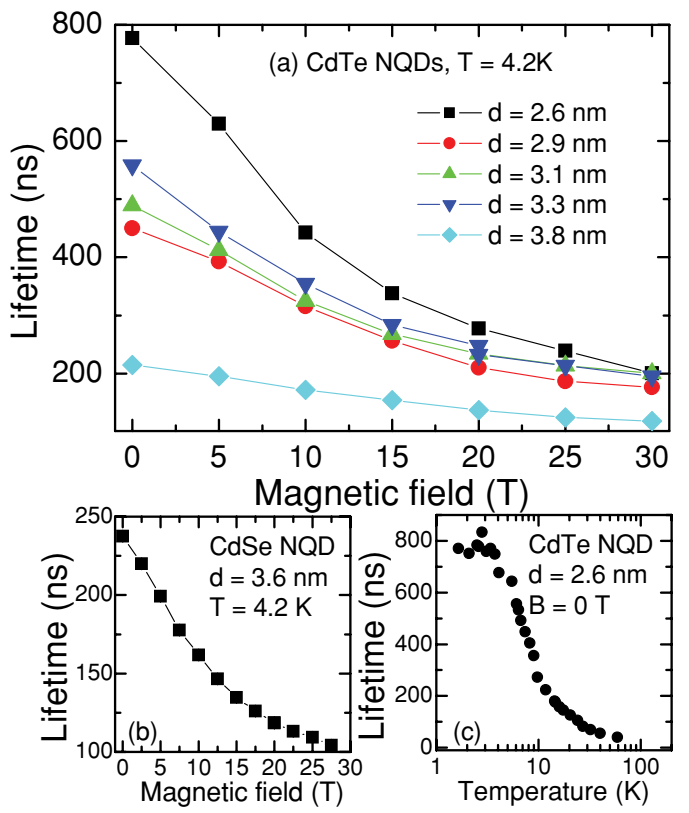

FIG. 3. (Color online) (a) Magnetic-field dependence of the exciton lifetimes of CdTe NQDs with different diameters at $T=$ 4.2 K. (b) Exciton lifetime versus magnetic field for a 3.6-nm diameter CdSe NQD. (c) Temperature dependence of the exciton lifetime of a 2.6-nm diameter CdTe NQD. The size of the symbols indicates the measurement error.

$\hbar \omega_{\mathrm{ST}}=(8 / 3 \pi)\left(a_{0} / a_{e x}\right)^{3} \varepsilon_{\text {exch }}$ for zinc-blende crystals and $\hbar \omega_{\mathrm{ST}}=(2 / \pi)\left(a_{0} / a_{e x}\right)^{3} \varepsilon_{\text {exch }}$ for wurtzite crystals, with bulk exciton radius $a_{e x}$, lattice constant $a_{0}$, the ratio of the light and heavy hole mass $\beta$, the crystal-anisotropy splitting $\Delta$, the exchange strength constant $\varepsilon_{\text {exch }}$, and the aspect ratio $\varepsilon$, defined as the ratio between the long and short axes of the NQDs. ${ }^{15}$

Spherical zinc-blende crystals with cubic symmetry have no crystal/shape anisotropy $\left(\Delta=\Delta_{\text {crystal }}+\Delta_{\text {shape }}=0\right)$. Nevertheless, the eight-fold degenerate ground state is split by the electron-hole exchange interaction, resulting in a five-fold degenerate, dark state, having $|F|=2\left(F_{m}= \pm 2\right.$, $F_{m}= \pm 1^{L}$, and $\left.F_{m}=0^{L}\right)$ and a three-fold degenerate, bright state, having $|F|=1\left(F_{m}= \pm 1^{U}\right.$ and $\left.F_{m}=0^{U}\right)$ [Fig. 4(a)]. The energy splitting between the levels increases with decreasing diameter, which is a general tendency caused by the enhanced exchange interaction in smaller NQDs. ${ }^{15}$

The intrinsic crystal anisotropy of the wurtzite crystal lattice leads to a modification of the exciton fine structure [Fig. 4(b)]. The crystal asymmetry along the NQD $c$ axis further splits the states, such that the ground state splits into five distinct states, of which the lowest two $\left(F_{m}= \pm 2\right)$ are optically dark and the two states at slightly higher energy $\left(F_{m}= \pm 1^{L}\right)$ are optically bright. The exciton fine structure also depends on the shape of the NQDs, as is clear from Fig. 4(c), which shows the size-dependent exciton levels for CdTe NQDs with aspect ratio $\varepsilon=1$.1. Most remarkably, this energy level structure is very similar to the levels of spherical CdSe NQDs in Fig. 4(b). Apart from a different size dependence of the $F_{m}=0^{L}$ level, the overall level structure is the same. In particular, the behavior of the two lowest exciton levels $\left(F_{m}= \pm 2\right.$ and $\left.F_{m}= \pm 1^{L}\right)$ is similar for spherical NQD having a wurtzite lattice and slightly elongated NQDs having a zinc-blende lattice.
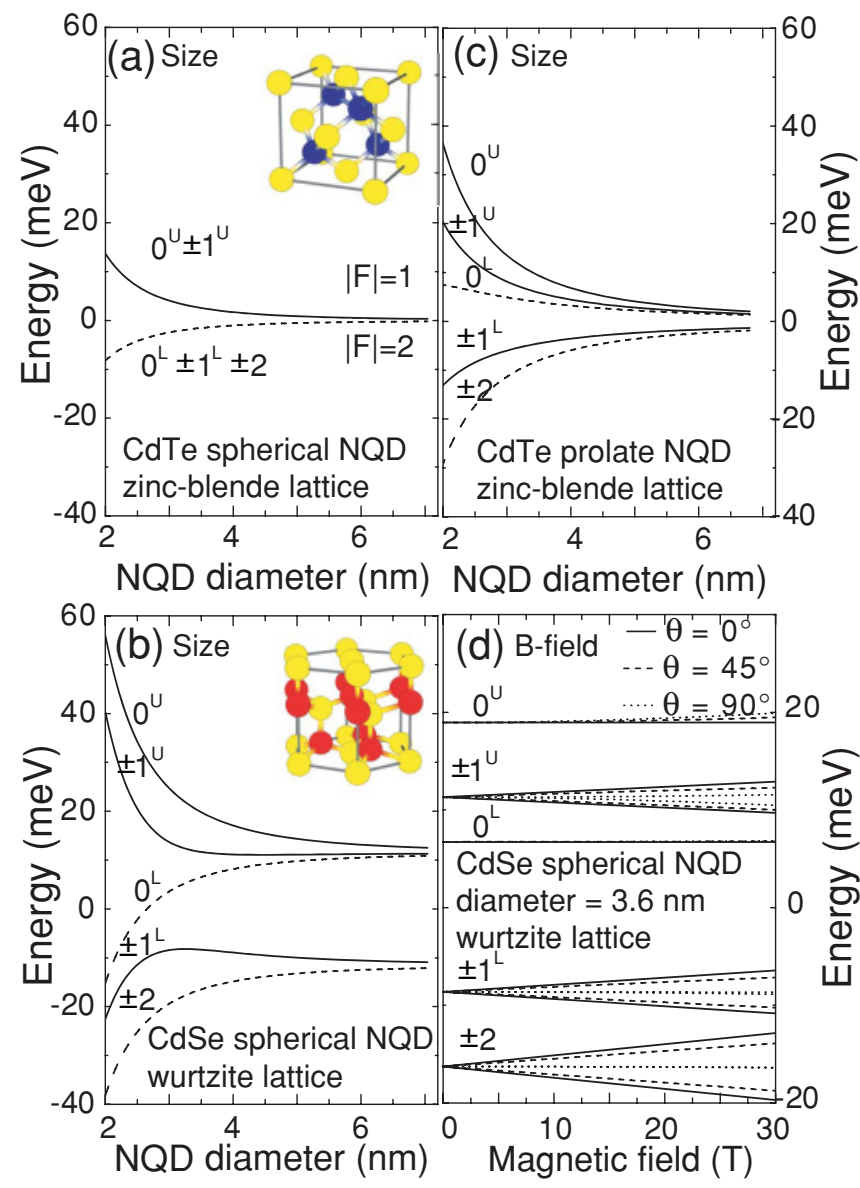

FIG. 4. (Color online) Exciton energy levels as function of the NQD size and the applied magnetic field. (a) Size dependence of spherical CdTe NQDs $\left(\hbar \omega_{\mathrm{ST}}=0.04 \mathrm{meV}, \beta=0.086, \Delta_{\text {crystal }}=0\right.$, and $a_{e x}=6.7 \mathrm{~nm}$ ). (b) Size dependence of spherical CdSe NQDs $\left(\hbar \omega_{\mathrm{ST}}=0.13 \mathrm{meV}, \beta=0.28, \Delta_{\text {crystal }}=25 \mathrm{meV}\right.$, and $\left.a_{e x}=5.6 \mathrm{~nm}\right)$. (c) Size dependence of a prolate CdTe NQD with an aspect ratio of $1.1\left(\hbar \omega_{\mathrm{ST}}=0.04 \mathrm{meV}, \beta=0.086, \Delta_{\text {crystal }}=0\right.$, and $\left.a_{e x}=6.7 \mathrm{~nm}\right)$. (d) Magnetic-field dependence of a spherical CdSe NQD with a diameter of $3.6 \mathrm{~nm}$ [parameters as in (b), $g_{e}=0.68$ and $g_{h}=-1.09$ ] (Ref. 15). $\theta$ is the angle between the $c$ axis of the crystal and the magnetic field. The insets of (a) and (b) show the unit cells of the crystal lattices of CdTe and CdSe. Solid (dashed) lines correspond to bright (dark) levels.

The magnetic-field dependence of such an exciton fine structure is shown in Fig. 4(d). Although this level structure is calculated using the parameters of a 3.6-nm-diameter spherical CdSe NQD, we assume that it is also approximately valid for a prolate CdTe NQD of similar size. The degenerate $F_{m}= \pm 2$ and $F_{m}= \pm 1^{U, L}$ exciton levels each split into two components as a result of the Zeeman effect. The splitting depends on the angle $\theta$ between the $c$ axis and the magnetic field and is maximal when the $c$ axis is parallel to the applied field $(\theta=0) .{ }^{15}$ An additional effect of the magnetic field is that it mixes the exciton states. The dark $F_{m}= \pm 2$ states mix with the bright $F_{m}=1^{L}$ states, which implies that the $F_{m}= \pm 2$ levels acquire oscillator strength in an applied magnetic field. This results in exciton lifetimes that depend on the strength of the magnetic field and its direction with respect to the $c$ axis 
of the NQD $(\theta)$ (Ref. 15):

$$
\frac{1}{\tau(B, \theta)}=\frac{\sqrt{1+\zeta^{2}+2 \zeta \cos \theta}-1-\zeta \cos \theta}{\sqrt{1+\zeta^{2}+2 \zeta \cos \theta}} \frac{3}{4 \tau_{\mathrm{rad}}},
$$

where $\tau_{\text {rad }}$ is a size-independent radiative lifetime, $\zeta=$ $\mu_{B} g_{\text {ex }} B / 3 \eta$ (with $\mu_{B}$ the Bohr magneton), $g_{\text {ex }}$ is the dark exciton $g$ factor, and $\eta=\left(a_{e x} / a\right)^{3} \hbar \omega_{\mathrm{ST}} \chi(\beta)$ is a factor that accounts for the size-dependent exchange splitting, using a dimensionless scaling factor $\chi(\beta)$, which can take values between 0.6 and $0.8 .^{10,15}$ This formula successfully describes the experimentally observed decreasing exciton lifetime with magnetic field ${ }^{11,23,24}$ of CdSe NQDs, and is also used to explain their field-induced circular polarization. ${ }^{11,24,25}$ In this regard, it is important to note that the lifetime of excitons in spherical CdTe NQDs is expected to be independent of the magnetic field: the $|F|=2$ and $|F|=1$ states of such zinc-blende NQDs do not mix, ${ }^{15}$ resulting in an exciton ground state that should be dark at all magnetic fields, in contrast to our experimental results.

Motivated by the predicted similarity of exciton fine structures of spherical CdSe and elongated CdTe NQDs [Figs. 4(b) and 4(c)], we use Eq. (1) to model the lifetimes of excitons in CdTe NQDs. The angle $\theta$ is now defined as the angle between the magnetic field and the elongated axis of the nanocrystal. To account for the finite exciton lifetime at $B=0$, we introduce a field-independent lifetime $\tau_{0}$ :

$$
\frac{1}{\tau}=\frac{1}{\tau_{0}}+\frac{1}{\tau(B)},
$$

where the value of $\tau_{0}$ is given by the experimental PL decay time at $B=0$, which equals 800,500 , and $200 \mathrm{~ns}$ for NDQs with $2.6,3.0$, and $3.8 \mathrm{~nm}$ diameters, respectively. This size dependence of the exciton lifetime is typical for both $\mathrm{CdSe}$ and CdTe systems. Longer lifetimes are observed at $4.2 \mathrm{~K}$ for decreasing NQD diameter. The size dependence of the lifetime is explained by an increase in the exchange splitting between the dark and bright states for smaller NQDs and by higher energies of acoustic phonon modes that enable the transition from the dark state. ${ }^{11,18-22,29}$ The $\tau_{0}$ has no effect on the magnetic-field dependence of the exciton lifetime determined by $\frac{1}{\tau(B)}$, which is obtained by the average over all angles $\theta$ between $0^{\circ}$ and $180^{\circ}$ :

$$
\frac{1}{\tau(B)}=\int_{0}^{\pi} \frac{1}{\tau(B, \theta)} \sin \theta d \theta .
$$

Figure 5 schematically shows the resulting exciton lifetimes as a function of magnetic field. The parameters used are $\hbar \omega_{\mathrm{ST}}=$ $0.04 \mathrm{meV}, a_{e x}=6.7 \mathrm{~nm}$, and $\chi(\beta)=0.61$, which were taken from the literature. ${ }^{15} \tau_{\text {rad }}$ and $g_{e x}$, the $g$ factor of the dark exciton, were used as adjustable parameters, leading to values $\tau_{\text {rad }}=10 \mathrm{~ns}$ and $\left|g_{\text {ex }}\right|=4$. The actual decrease of the PL decay time with $B$ is entirely determined by the value of $\zeta$ [cf. Eq. (1)]; so, in our case, this is determined by the free parameter $g_{e x}$. The value found for $g_{e x}$ is larger than that reported in the literature, ${ }^{24,30,31}$ but close to the theoretically expected value $\left|g_{e x}\right| \approx 4$ for the dark exciton ground state. ${ }^{15}$

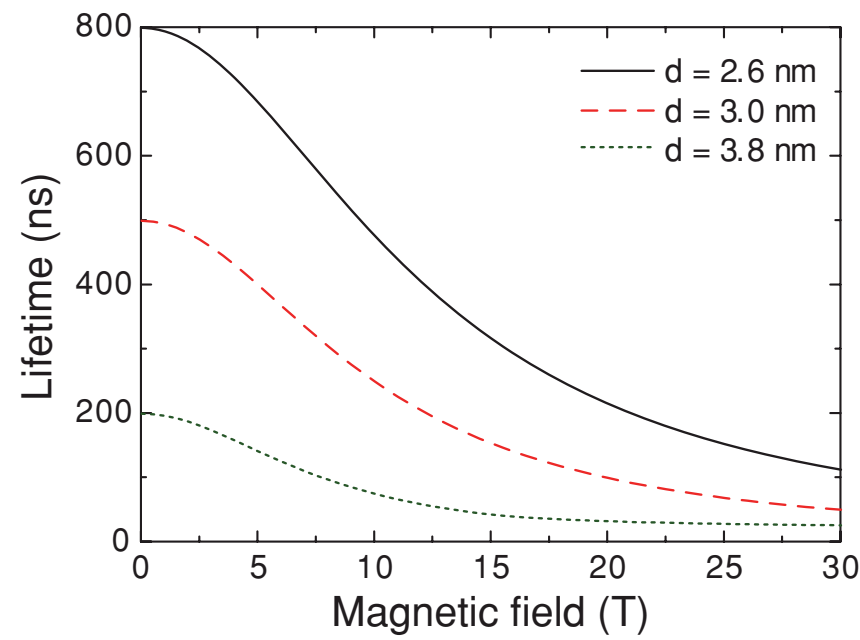

FIG. 5. (Color online) Modeled exciton lifetimes as a function of the applied magnetic field for CdTe NQDs with three different diameters. The model is described in the main text. The following parameters have been used: $\tau_{\text {rad }}=10 \mathrm{~ns}, \hbar \omega_{\mathrm{ST}}=0.04 \mathrm{meV}$, $a_{e x}=6.7 \mathrm{~nm}, \chi(\beta)=0.61$, and $\left|g_{e x}\right|=4$.

Figure 5 demonstrates that this model qualitatively explains the behavior of the PL decay times in CdTe NQDs as a function of the magnetic field. Although our model is very schematic and ignores some processes contributing to the PL decay, such as relaxation to surface states ${ }^{25}$ and the influence of surface ligands ${ }^{32}$ and phonon-assisted transitions, ${ }^{29}$ we believe that it catches the essential physics of the $B$-dependent exciton PL decay times. Our results strongly suggest that the mechanism for the reduced lifetimes of exciton in CdTe NQDs in an applied magnetic field is the same as that in CdSe NQDs. We attribute this similarity to the nonspherical shape of the zinc-blende NQDs, which results in a NQD symmetry that is equal to that of wurzite NQDs with a spherical shape.

\section{CONCLUSIONS}

In conclusion, we have shown that the PL decay time of CdTe NQDs decreases when their size is increased and when an external magnetic field is applied. The decrease of the PL decay time of a NQD in a magnetic field is caused by the magneticfield-induced mixing of dark and bright exciton states. The latter phenomenon can only be explained when a slight shape asymmetry of the CdTe NQDs is assumed. These results show that it is not possible to distinguish the exciton fine structure of a spherical NQD having a wurtzite lattice from a slightly elongated NQD having a zinc-blende lattice.

\section{ACKNOWLEDGMENTS}

The authors thank R. Plantenga for the temperaturedependent lifetime measurements. This work was supported by EuroMagNET II under the EU Contract No. 228043, and by the Stichting voor Fundamenteel Onderzoek der Materie (FOM), and financially supported by the Nederlandse Organisatie voor Wetenschappelijk Onderzoek (NWO). 
*p.christianen@science.ru.nl

${ }^{1}$ A. P. Alivisatos, Science 271, 933 (1996).

${ }^{2}$ C. de Mello Donegá, Chem. Soc. Rev., doi:10.1039/c0cs00055h.

${ }^{3}$ L. Qu and X. Peng, J. Am. Chem. Soc. 124, 2049 (2002).

${ }^{4}$ P. T. K. Chin, C. de Mello Donegá, S. S. van Bavel, S. C. J. Meskers, N. A. J. M. Sommerdijk, and R. A. J. Janssen, J. Am. Chem. Soc. 129, 14880 (2007).

${ }^{5}$ D. V. Talapin, J.-S. Lee, M. V. Kovalenko, and E. V. Shevchenko, Chem. Rev. 110, 389 (2010).

${ }^{6}$ S. Coe, W.-K. Woo, M. Bawendi, and V. Bulovic, Nature (London) 420, 800 (2002).

${ }^{7}$ N. Tessler, V. Medvedev, M. Kazes, S. Kan, and U. Banin, Science 295, 1506 (2002).

${ }^{8}$ A. P. Alivisatos, Nat. Biotechnol. 22, 47 (2004).

${ }^{9}$ A. J. Nozik, Chem. Phys. Lett. 457, 3 (2008).

${ }^{10}$ A. L. Efros and M. Rosen, Annu. Rev. Mater. Sci. 30, 475 (2000).

${ }^{11}$ M. Furis, J. A. Hollingsworth, V. Klimov, and S. Crooker, J. Phys. Chem. B 109, 15332 (2005).

${ }^{12}$ V. L. Colvin, M. C. Schlamp, and A. P. Alivisatos, Nature (London) 370, 354 (1994).

${ }^{13}$ B. L. Wehrenberg, C. Wang, and P. Guyot-Sionnest, J. Phys. Chem. B 106, 10634 (2002).

${ }^{14}$ A. Shavel, N. Gaponik, and A. Eychmuller, J. Phys. Chem. B 108, 5905 (2004).

${ }^{15}$ A. L. Efros, M. Rosen, M. Kuno, M. Nirmal, D. J. Norris, and M. Bawendi, Phys. Rev. B 54, 4843 (1996).

${ }^{16}$ J. M. An, A. Franceschetti, and A. Zunger, Nano. Lett. 7, 2129 (2007).

${ }^{17}$ D. J. Norris, A. L. Efros, M. Rosen, and M. G. Bawendi, Phys. Rev. B 53, 16347 (1996).
${ }^{18}$ S. A. Crooker, T. Barrick, J. A. Hollingsworth, and V. I. Klimov, Appl. Phys. Lett. 82, 2793 (2003).

${ }^{19}$ A. F. van Driel, G. Allan, C. Delerue, P. Lodahl, W. L. Vos, and D. Vanmaekelbergh, Phys. Rev. Lett. 95, 236804 (2005).

${ }^{20}$ S. Neeleshwar, C. L. Chen, C. B. Tsai, Y. Y. Chen, C. C. Chen, S. G. Shyu, and M. S. Seehra, Phys. Rev. B 71, 201307 (2005).

${ }^{21}$ C. de Mello Donegá, M. Bode, and A. Meijerink, Phys. Rev. B 74, 085320 (2006).

${ }^{22}$ C. de Mello Donegá and R. Koole, J. Phys. Chem. C 113, 6511 (2009).

${ }^{23}$ M. Nirmal, D. J. Norris, M. Kuno, M. G. Bawendi, A. L. Efros, and M. Rosen, Phys. Rev. Lett. 75, 3728 (1995).

${ }^{24}$ E. Johnston-Halperin, D. D. Awschalom, S. A. Crooker, A. L. Efros, M. Rosen, X. Peng, and A. P. Alivisatos, Phys. Rev. B 63, 205309 (2001).

${ }^{25}$ F. J. P. Wijnen, J. H. Blokland, P. T. K. Chin, P. C. M. Christianen, and J. C. Maan, Phys. Rev. B 78, 235318 (2008).

${ }^{26}$ R. D. Schaller, S. A. Crooker, D. A. Bussian, J. M. Pietryga, J. Joo, and V. I. Klimov, Phys. Rev. Lett. 105, 067403 (2010).

${ }^{27}$ S. F. Wuister, I. Swart, F. van Driel, S. G. Hickey, and C. de Mello Donegá, Nano Lett. 3, 503 (2003).

${ }^{28}$ S. F. Wuister, C. de Mello Donegá, and A. Meijerink, J. Am. Chem. Soc. 126, 10397 (2004).

${ }^{29}$ D. Oron, A. Aharoni, C. de Mello Donegá, J. van Rijssel, A. Meijerink, and U. Banin, Phys. Rev. Lett. 102, 177402 (2009).

${ }^{30}$ J. A. Gupta, D. D. Awschalom, A. L. Efros, and A. V. Rodina, Phys. Rev. B 66, 125307 (2002).

${ }^{31}$ E. Lifshitz, L. Fradkin, A. Glozman, and L. Langof, Annu. Rev. Phys. Chem. 55, 509 (2004).

${ }^{32}$ P. Guyot-Sionnest, B. Wehrenberg, and D. Yu, J. Chem. Phys. 123, 074709 (2005). 